\title{
THE VIBRATION AND STABILITY OF A FRICTION-GUIDED, TRANSLATING STRING
}

\author{
S.-P. Cheng and N. C. Perkins \\ Department of Mechanical Engineering and Applied Mechanics, University of Michigan, \\ Ann Arbor, Michigan 48109-2125, U.S.A.
}

(Received 10 November 1989 and in revised form 19 March 1990)

\begin{abstract}
Eyelets, capstans and cylindrical surfaces are often used in thread, fiber and paper handling machinery to guide the axially moving element. In addition to providing positional control, these "guides" introduce dry friction forces that alter the vibration and stability characteristics of the system. This paper examines the lateral response of a string that slides through an elastically supported, dry friction guide. Exact expressions are derived for the linear response under free and forced conditions. Solutions for the eigenvalue spectrum exhibit unusual features including multiple divergence instabilities, regions of flutter instability, and regions of curve veering associated with mode localization. A second order perturbation solution is derived to examine the behavior of the eigenvalue spectrum in regions of flutter instability and curve veering. The analysis highlights the common features of these two phenomena and suggests ways to minimize vibration by adjusting various design variables. The analysis also demonstrates that the eigenvalue loci in regions of flutter instability and curve veering are naturally described by a local hyperbolic approximation.
\end{abstract}

\section{INTRODUCTION}

A large class of mechanical systems, commonly referred to as axially moving materials [1], employs a translating element as a mechanism for transmitting power, material or information. The translating string is an important model for flexible, axially moving materials such as translating belts, chains, fibers, magnetic tapes, paper and threads.

The stability of a translating string is limited by a critical translation speed; above this speed a buckling instability occurs [2]. The critical speed remains unaltered when the translating string is coupled to subsystems that do not affect the system tension such as elastic guides $[3,4]$ and elastic foundations $[5,6]$. Any distributed elastic foundation, however, renders the translating string model dispersive, and significant attenuation of vibration amplitudes is possible by the proper selection of the foundation stiffness and the translation speed [6]. Pulley or wheel subsystems mounted on compliant supports lead to a speed-tension relation that can alter the critical speed $[7,8]$.

In contrast to the above studies, which feature conservative subsystems, very little research has addressed the response of axially moving materials coupled to non-conservative subsystems. In many applications, the tautly drawn element slides across fixed guiding surfaces such as capstans, cylinders, eyelets, record/erase heads and rollers, which may generate substantial dry friction forces. Dry friction forces are responsible for the inadvertent twisting of yarn in contact with rollers [9], the motion of threads along cylindrical surfaces [10], and the self-excitation of longitudinal vibrations in magnetic tapes [11]. Despite the pervasive use of these dry friction guides, there have been no studies of the effect of dry friction on the lateral response of axially moving materials. 
The purpose of the study reported here is to investigate the dynamic response of an axially moving material that is in contact with a dry friction guide. A theoretical model is derived that governs the lateral motion of a string which slides through an elastically supported, dry friction guide. Exact methods are used to derive closed form expressions for the linear response of the string/guide system under free and forced conditions. The solutions illustrate how the vibration and stability of the string/guide system can be modified by changing design variables.

\section{EQUATIONS OF MOTION}

The system depicted in Figure 1 consists of a flexible string that is drawn to the right between two eyelets at $X=0$ and $X=L$. The eyelet at $X=L$ remains stationary while the eyelet at $X=0$ may execute a prescribed lateral displacement $F(T)$. The string, which translates with speed $S$, slides through an elastically supported guide with negligible mass at $X=D$. The top and bottom surfaces of the guide are assumed to remain in contact with the string due to the relatively large static forces $N$ that are applied externally through two elastic supports $K_{1}$ and $K_{2}$. As the string translates, dry friction forces $F_{1}$ and $F_{2}$ develop on the surfaces of the guide and create a non-uniform tension $R(X, T)$ along the string.

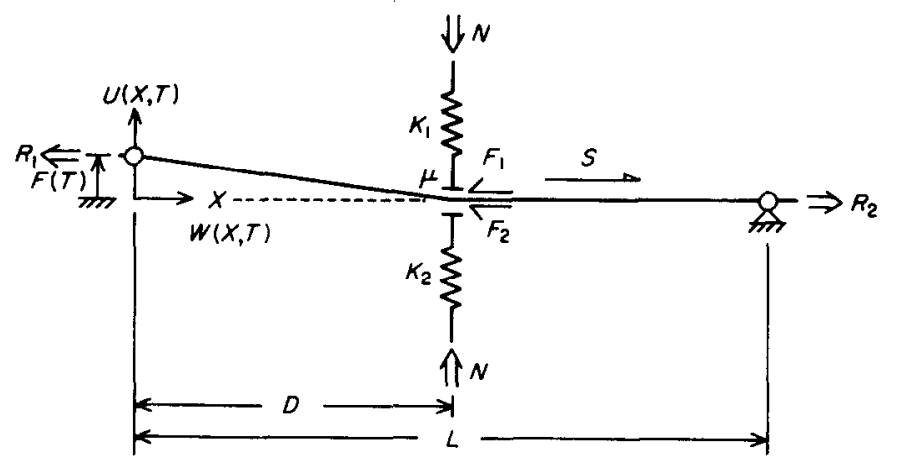

Figure 1. Translating string/guide system. String is drawn through two eyelets and a dry friction guide by constant downstream tension $\boldsymbol{R}_{\mathbf{2}}$. Guide surfaces remain in contact with the string and are preloaded by a large, static force $N$.

With the elementary assumptions of the moving threadline model [2] and the inclusion of the strain energy and kinetic energy due to small extensions, Hamilton's principle becomes

$$
\begin{gathered}
\int_{T_{1}}^{T_{2}}\left(\delta \frac { 1 } { 2 } \left[\int_{0}^{L}\left\{\rho\left(S+W_{, T}+S W_{, X}\right)^{2}+\rho\left(U_{, T}+S U_{, X}\right)^{2}-R(X, T) U_{, X}^{2}-E A W_{, X}^{2}\right\} \mathrm{d} X\right.\right. \\
\left.\left.-\left(K_{1}+K_{2}\right) U(D, T)^{2}\right]-\left(F_{1}+F_{2}\right) \delta W(D, T)\right) \mathrm{d} T=0 .
\end{gathered}
$$

In equation (1), $U(X, T)$ and $W(X, T)$ represent string displacements in the transverse and longitudinal directions, respectively, $\rho$ is the string mass/length, and $E A$ is the string section stiffness. Taking the first variation in equation (1) leads to the equations of planar motion

$$
\begin{gathered}
{\left[\left(R-\rho S^{2}\right) U_{, X}\right]_{, X}=\rho U_{, T T}+2 \rho S U_{, X T}+\left(K_{1}+K_{2}\right) U \delta(X-D)} \\
\left(E A-\rho S^{2}\right) W_{, X X}=\rho W_{, T T}+2 \rho S W_{, X T}+\left(F_{1}+F_{2}\right) \delta(X-D)
\end{gathered}
$$


where $\delta(X-D)$ denotes the Dirac delta distribution at $X=D$. The string tension is written as $R=R_{1}+\left(R_{2}-R_{1}\right) H(X-D)$, where $R_{1}(X, T)$ and $R_{2}(X, T)$ represent the tension in the string segments upstream and downstream of the guide, respectively. Note that the friction forces generated by the guide depend on the transverse string displacement and are given by

$$
F_{1}=\mu\left[N+K_{1} U(D, T)\right], \quad F_{2}=\mu\left[N-K_{2} U(D, T)\right],
$$

where $\mu$ is the coefficient of friction between the guide and the string.

The model is simplified by noting that the speed of propagation of longitudinal waves in the moving string, $\sqrt{E A / \rho}$, greatly exceeds the speed of propagation of transverse waves, $\sqrt{R / \rho}$. Thus, in the time scale of transverse motion, the string stretches in a quasi-static manner and the inertia terms $\rho\left[W_{, T T}+2 S W_{, X T}+S^{2} W_{, X X}\right]$ may be neglected. Integrating equation (3) in this case shows that the upstream tension $R_{1}$ reduces to a function of time alone,

$$
R_{1}=R_{2}-\left(F_{1}+F_{2}\right)=R_{2}-\mu\left[2 N+\left(K_{1}-K_{2}\right) U(D, T)\right],
$$

where the constitutive relation $R=E A W_{, X}$ and equations (4) and (5) have been used and $R_{2}$ is the prescribed downstream tension. With $U(X, T)=U_{1}$ for $0<X<D$ and $U(X, T)=U_{2}$ for $D<X<L$, the equation of transverse motion (2) becomes:

$$
\left(R_{1}-\rho S^{2}\right) U_{1, X X}=\rho U_{1, T T}+2 \rho S U_{1, X T}, \quad\left(R_{2}-\rho S^{2}\right) U_{2, X X}=\rho U_{2, T T}+2 \rho S U_{2, x T},
$$

with boundary conditions

$$
\begin{gathered}
U_{1}(D, T)-U_{2}(D, T)=0, \\
\left(R_{2}-\rho S^{2}\right) U_{2, x}(D, T)-\left(R_{1}-\rho S^{2}\right) U_{1, X}(D, T)-\left(K_{1}+K_{2}\right) U(D, T)=0, \\
U_{1}(0, T)=F(T), \quad U_{2}(L, T)=0 .
\end{gathered}
$$

Note that equations (7) and (10) are non-linear due to the dependence of the upstream tension on the transverse motion of the string; see equation (6). This interesting non-linear coupling represents a possible source of internal resonance and is the subject of current research. The present study is focused on the related linear problem that results when equal support stiffness $K_{1}=K_{2}=K$ is assumed. In this case, the tension $R_{1}$ is constant.

\section{FREE, LINEAR RESPONSE}

\subsection{CHARACTERISTIC EQUATION}

Exact methods can be used to examine the linear vibration and stability of the string/guide system for the case of free response, $F(T)=0$ in equation (11). Using the separable solutions $U_{j}(X, T)=V_{j}(X) \mathrm{e}^{i \Omega T}, j=1,2$, in the homogeneous equations (7)(11) leads to the (non-dimensional) eigenvalue problem

$$
\begin{gathered}
\left(\gamma^{2}-\eta^{2}\right) v_{1}^{\prime \prime}-\mathrm{i} 2 \eta \omega v_{1}^{\prime}+\omega^{2} v_{1}=0, \quad 0<x<d, \\
\left(1-\eta^{2}\right) v_{2}^{\prime \prime}-\mathrm{i} 2 \eta \omega v_{2}^{\prime}+\omega^{2} v_{2}=0, \quad d<x<1, \\
v_{1}(0)=0, \quad v_{2}(1)=0, \\
v_{1}(d)-v_{2}(d)=0, \quad\left(1-\eta^{2}\right) v_{2}^{\prime}(d)-\left(\gamma^{2}-\eta^{2}\right) v_{1}^{\prime}(d)-k v_{1}(d)=0 .
\end{gathered}
$$

The non-dimensional quantities introduced here are

$$
\begin{aligned}
& x=X / L, \quad v_{1}=V_{1} / L, \quad v_{2}=V_{2} / L, \quad d=D / L, \quad c^{2}=R_{2} / \rho, \\
& \gamma^{2}=R_{1} / R_{2}, \quad \eta=S / c, \quad t=T c / L, \quad \omega=\Omega L / c, \quad k=2 K L / \rho c^{2} .
\end{aligned}
$$


The solutions

$$
v_{1}(x)=A_{1} \mathrm{e}^{\mathrm{i} \phi_{1} x}\left[\sin \left(\psi_{1} x\right)\right], \quad v_{2}(x)=A_{2} \mathrm{e}^{\mathrm{i} \phi_{2} x}\left[\sin \left(\psi_{2} x\right)-\tan \left(\psi_{2}\right) \cos \left(\psi_{2} x\right)\right],
$$

which contain two arbitrary constants $A_{1}$ and $A_{2}$, satisfy the field equations (12) and (13) and the two boundary conditions (14), provided that

$$
\phi_{1}=\eta \omega /\left(\gamma^{2}-\eta^{2}\right), \quad \phi_{2}=\eta \omega /\left(1-\eta^{2}\right), \quad \psi_{1}=\gamma \omega /\left(\gamma^{2}-\eta^{2}\right), \quad \psi_{2}=\omega /\left(1-\eta^{2}\right) .
$$

Applying the remaining boundary conditions (15) and simplifying the result leads to the characteristic equation

$$
\begin{aligned}
C(\omega)= & k \sin \left(\psi_{1} d\right) \sin \left[\psi_{2}(1-d)\right]+\omega \sin \left(\psi_{1} d\right) \cos \left[\psi_{2}(1-d)\right] \\
& +\gamma \omega \cos \left(\psi_{1} d\right) \sin \left[\psi_{2}(1-d)\right]=0 .
\end{aligned}
$$

The eigenvalues $\omega_{l}, l=1,2,3, \ldots$, governing the natural frequency and stability of each vibration mode are determined as the roots of equation (20) for prescribed values of the string translation speed $\eta$, guide position $d$, stiffness $k$, and upstream tension $\gamma$. The quantity $\gamma$ in equations (16) may also be interpreted as the ratio of the sound speeds of transverse waves in the upstream and downstream portions of the string, and it is related to the applied pre-load $N$ and friction $\mu$ of equation (6). For the special case $\gamma=1$ $(\mu=0)$, equation (20) reduces to the characteristic equation for a class of frictionless systems studied in reference [6].

\subsection{EigenVAlue SPECTRUM}

As noted above, the eigenvalue spectrum for the string/guide system may be determined numerically for a given set of parameters. The first example illustrates how this spectrum depends on the guide stiffness and translation speed for a centrally located guide $(d=0 \cdot 5)$ with constant friction $(\gamma=0.5)$. In Figures 2 and 3 are shown the eigenvalues as functions of translation speed $\eta$ for representative values of the guide stiffness $k$. The real parts (i.e., natural frequencies) and the positive imaginary parts of the complex eigenvalues are plotted separately over the range of speeds $0<\eta<1$.

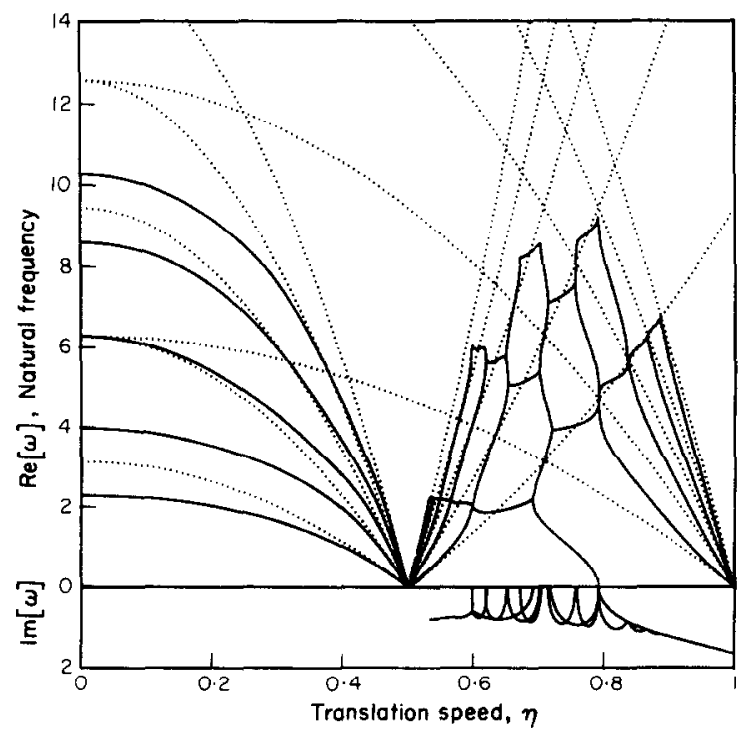

Figure 2. Eigenvalue spectrum for limiting cases of guide stiffness. Real and (positive) imaginary parts of eigenvalues are plotted versus translation speed $\eta$ for the case of a central guide $(d=0 \cdot 5)$ with constant friction $(y=0.5)$. Solid curves are the solution for $k=0$; dotted curves are the solution for $k \rightarrow \infty$. 
For the limiting case of infinite guide stiffness, $k \rightarrow \infty$, the upstream and downstream string segments become decoupled, and the characteristic equation (20) reduces to

$$
f(\omega, \eta) g(\omega, \eta)=0,
$$

where

$$
f(\omega, \eta)=\sin \left(\psi_{1} d\right), \quad g(\omega, \eta)=\sin \left[\psi_{2}(1-d)\right] .
$$

The functions $f$ and $g$, which are the characteristic equations for upstream and downstream string segments with fixed ends, have real roots:

$$
\omega_{m}=\frac{m \pi\left(\gamma^{2}-\eta^{2}\right)}{\gamma d}, \quad m= \pm 1, \pm 2, \pm 3 \ldots
$$

and

$$
\omega_{n}=\frac{n \pi\left(1-\eta^{2}\right)}{1-d}, \quad n= \pm 1, \pm 2, \pm 3 \ldots,
$$

respectively. These solutions give the natural frequencies of two classical threadlines [2], one of length $d$ with sound speed $\gamma$, and the other of length $1-d$ with sound speed 1 . The dotted curves in Figure 2 illustrate the two families of frequency speed loci given by expressions (24) and (25). This elementary solution exhibits two critical speeds, $\gamma$ and 1. The first critical speed, $\eta=\gamma$, leads to a buckling instability in the relatively slack upstream segment. For speeds $\gamma<\eta<1$, the upstream and downstream segments remain stable according to the present linear analysis. However, the contribution of geometric non-linearities increases with translation speed [7], and this solution is interpreted as a first approximation only. Indeed, as described below, the string/guide system becomes unstable in the range $\gamma<\eta<1$ for a guide with finite stiffness.

The first five eigenvalues for the extreme case of vanishing guide stiffness, $k=0$ (solid curves) are also depicted in Figure 2. Note that altering the guide stiffness does not alter the system tension, and the critical speeds, $\eta=\gamma$ and $\eta=1$, are unaffected. For translation speeds greater than $\gamma$, regions exist in which pairs of eigenvalues become complex conjugates; in such cases, the non-conservative friction forces lead to flutter instability.

The transition of the eigenvalue spectrum between the limiting cases represented in Figurc 2 is depicted in Figurc 3, where an intermediate value of the guide stiffness, $k-20$, is considered (solid curves). In Figure 3, an important comparison is made with the spectrum for the decoupled system, $k \rightarrow \infty$ (dotted curves). The crossings (i.e., repeated frequencies) of the frequency speed loci for the decoupled system mark regions of abrupt change in the spectrum for finite stiffness case, $k=20$. Below the first critical speed in Figure 3, the loci crossings for the decoupled case become regions of curve veering for the finite stiffness case; for example, see the boxed region to the left in Figure 3 . The term curve veering is commonly used to describe eigenvalue loci that abruptly turn away from each other as their separation decreases [4].

The significance of this result is that the modes of vibration associated with curve veering are highly localized to either the upstream or downstream segments. Localized vibrations in non-gyroscopic systems have recently been studied in detail; see, for example, reference [12]. In Figure 4 are depicted the third and fourth modes at the translation speeds $\eta=0.28$ and 0.37 , which mark the beginning and end of the region of curve veering shown by the left-hand box in Figure 3. The eigenfunctions are, in general, complex, and the curves in Figure 4 are the envelope of the modal response; see the square-bracketed functions in equations (17) and (18). Note that while the third mode is localized to the downstream portion in the beginning of the veering region, it becomes 


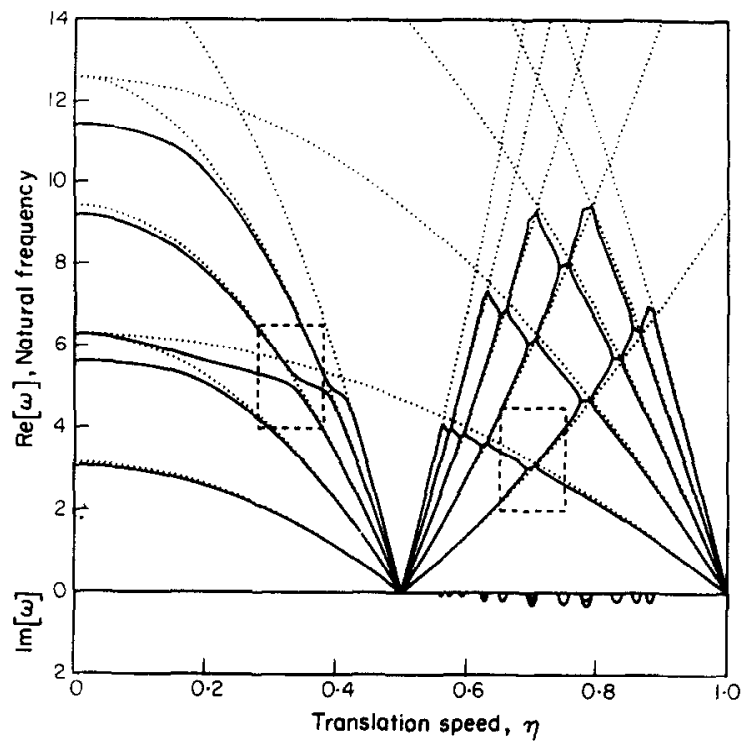

Figure 3. Eigenvalue spectrum for intermediate guide stiffness. Real and (positive) imaginary parts of eigenvalues are plotted versus translation speed $\eta$ for case of a central guide $(d=0 \cdot 5)$ with constant friction $(\gamma=0 \cdot 5)$. Solid curves are the solution for $k=20$; dotted curves are the solution for $k \rightarrow \infty$. Boxes indicate representative regions of curve veering and flutter instability.

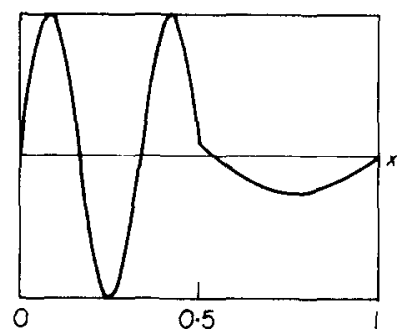

(a)

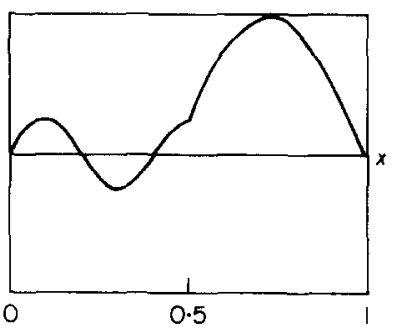

(c)

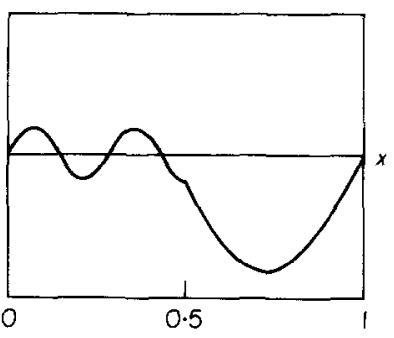

(b)

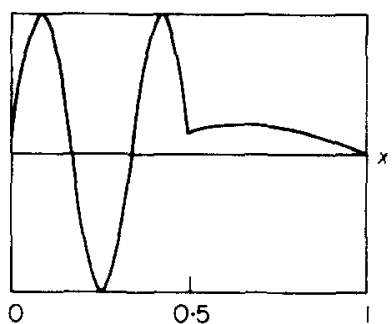

(d)

Figure 4. Localization of free response. Envelopes of third and fourth modes are shown at $\eta=0 \cdot 28$ and $\eta=0.37$; refer to boxed region at left in Figure 3 . (a) Mode 4, $\eta=0 \cdot 28$; (b) mode $4, \eta=0.37$; (c) mode 3 , $\eta=0.28$; (d) mode $3, \eta=0.37$. 
localized to the upstream portion at the end of the region. The opposite conclusion holds for the fourth mode. Thus, small changes in the translation speed produce large changes in the magnitude of the modal response in the upstream and downstream segments.

The loci crossings in the decoupled system $(k \rightarrow \infty)$ above the first critical speed in Figure 3 indicate regions of flutter instability in the coupled system $(k=20)$. Note that the regions of flutter instability of various mode pairs are dense in the interval $\gamma<\eta<1$, and there are no speeds at which all the modes are stable. This instability region grows with increasing friction, which, in turn, reduces the upstream tension $\gamma$.

A second example considers how the eigenvalue spectrum depends on guide location. The spectrum is shown in Figure 5 as a function of guide location $d$ for constant translation speed $\eta=0.3$ and friction $\gamma=0.5$. The dotted curves denote the natural frequencies for the decoupled system $(k \rightarrow \infty)$ and the solid curves denote those of a coupled system $(k=20)$. For the subcritical case $(\eta<\gamma)$, the loci crossing for the decoupled system again evolve into regions of curve veering for the coupled system. Within these regions, the associated eigenfunctions are extremely sensitive to small changes in guide location.

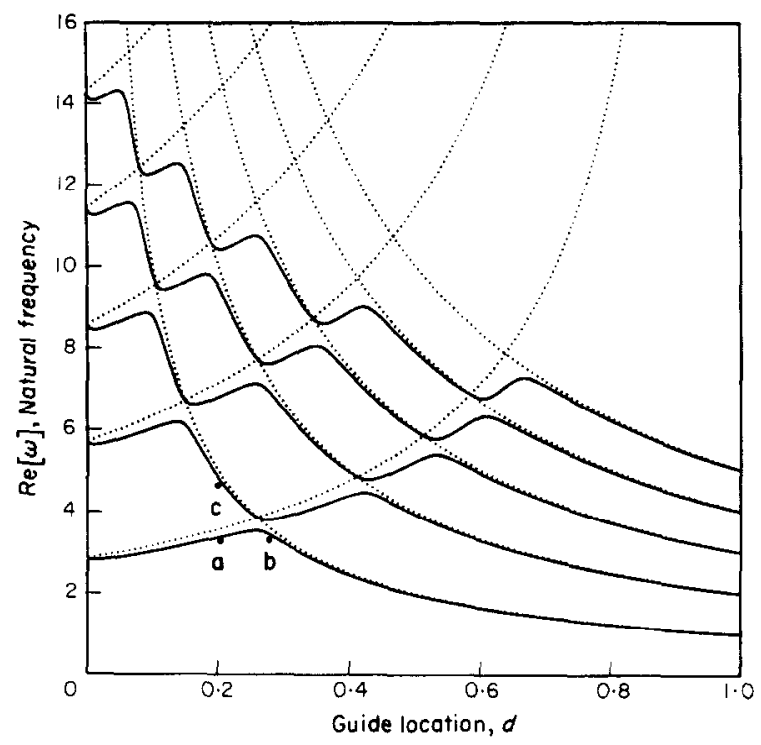

Figure 5. Influence of guide location on eigenvalue spectrum. Real parts of eigenvalues are plotted versus guide location $d$ for the subcritical case $\eta=0.3, \gamma=0.5$. Solid curves are the solution for $k=20$; dotted curves are the solution for $k \rightarrow \infty$.

\subsection{DEVELOPMENT OF REGIONS OF CURVE VEERING AND FLUTTER INSTABILITY}

From the above examples, it is clear that loci crossings in the decoupled system may evolve into regions of curve veering or regions of flutter instability. One might, therefore, suspect some strong connection in the development of these two phenomena. This connection, in the present problem, is examined by the following perturbation analysis, which provides an accurate, closed form approximation of the eigenvalue spectrum in these regions.

Several perturbation analyses have been utilized for eigenvalue problems exhibiting curve veering; see the discussion in reference [13]. In the present problem, a perturbation analysis of the characteristic equation (20) is employed to highlight how regions of curve veering and flutter instability can both be treated in a single analysis. 
First, the characteristic equation (20) is rewritten as

$$
C(\omega, \eta) / k=f(\omega, \eta) g(\omega, \eta)+\varepsilon h(\omega, \eta)=0,
$$

where

$$
h(\omega, \eta)=\omega \sin \left(\psi_{1} d\right) \cos \left[\psi_{2}(1-d)\right]+\gamma \omega \cos \left(\psi_{1} d\right) \sin \left[\psi_{2}(1-d)\right],
$$

and $\varepsilon=1 / k$ is termed the coupling factor, in keeping with reference [13]. The functions $f$ and $g$ retain their former definitions, given by equations (22) and (23), and the function $h$ represents the characteristic equation of the fully coupled system $(\varepsilon \rightarrow \infty)$. Although in the present formulation the dependence of the eigenvalue $\omega$ on the translation speed $\eta$ is examined, the dependence on any other parameter (e.g., $k, d$ or $\gamma$ ) could be examined in a similar manner. An approximation to equation (26) is derived that is valid to second order in $\varepsilon$ in a small neighborhood about a loci crossing point. The locations of the loci crossing points in Figure 3, denoted by $\left(\omega^{*}, \eta^{*}\right)$, are determined as the common solutions of equations (24) and (25). These common natural frequencies of the decoupled subsystems, $f\left(\omega^{*}, \eta^{*}\right)=0$ and $g\left(\omega^{*}, \eta^{*}\right)=0$, are, of course, natural frequencies of the coupled system, $C\left(\omega^{*}, \eta^{*}\right)=0$. Thus, the frequency speed loci of the coupled system must always pass through the loci crossing points regardless of the degree of coupling [3]. Note that $h(\omega, \eta)$ can be rewritten as

$$
h(\omega, \eta)=p(\omega, \eta) f(\omega, \eta)+q(\omega, \eta) g(\omega, \eta),
$$

in which the functions $f$ and $g$ are weighted by

$$
p(\omega, \eta)=\omega \cos \left[\psi_{2}(1-d)\right], \quad q(\omega, \eta)=\gamma \omega \cos \left(\psi_{1} d\right) .
$$

The approximation is achieved by considering first order perturbations of both $\omega$ and $\eta$, given by $\omega=\omega^{*}+\varepsilon \bar{\omega}, \eta=\eta^{*}+\varepsilon \bar{\eta}$, where the small coupling $\varepsilon$ represents the perturbation parameter. Expanding the functions $f, g$ and $h$ in first order Taylor series and substituting into (26) yields

$$
\left(a_{1} \bar{\omega}+b_{1} \bar{\eta}\right)\left(a_{2} \bar{\omega}+b_{2} \bar{\eta}\right)+\left[\left(p^{*} a_{1}+q^{*} a_{2}\right) \bar{\omega}+\left(p^{*} b_{1}+q^{*} b_{2}\right) \bar{\eta}\right]=0
$$

where

$$
\begin{array}{cc}
a_{1}=\left.(\partial f / \partial \omega)\right|_{\left(\omega^{*}, \eta^{*}\right)}, \quad a_{2}=\left.(\partial g / \partial \omega)\right|_{\left(\omega^{*}, \eta^{*}\right)}, & b_{1}=\left.(\partial f / \partial \eta)\right|_{\left(\omega^{*}, \eta^{*}\right),} \\
b_{2}=\left.(\partial g / \partial \eta)\right|_{\left(\omega^{*}, \eta^{*}\right)}, \quad p^{*}=p\left(\omega^{*}, \eta^{*}\right), & q^{*}=q\left(\omega^{*}, \eta^{*}\right) .
\end{array}
$$

Note that the quantity $\Delta=\left(a_{1} b_{2}-a_{2} b_{1}\right)^{2} \geqslant 0$; thus, the approximate characteristic equation (31) represents a general hyperbola in the $(\bar{\omega}, \bar{\eta})$ plane near the loci crossing $\left(\omega^{*}, \eta^{*}\right)$. Letting $\left(\bar{\omega}=\omega_{1}, \bar{\eta}=\eta_{1}\right)$ denote the intersection of the asymptotes of the hyperbola enables the approximation to be rewritten as

$$
\left[a_{1}\left(\bar{\omega}-\omega_{1}\right)+b_{1}\left(\bar{\eta}-\eta_{1}\right)\right]\left[a_{2}\left(\bar{\omega}-\omega_{1}\right)+b_{2}\left(\bar{\eta}-\eta_{1}\right)\right]=\Lambda,
$$

where

$$
\omega_{1}=\left(p^{*} b_{1}-q^{*} b_{2}\right) /\left(a_{1} b_{2}-a_{2} b_{1}\right), \quad \eta_{1}=\left(q^{*} a_{2}-p^{*} a_{1}\right) /\left(a_{1} b_{2}-a_{2} b_{1}\right), \quad \Lambda=p^{*} q^{*} .
$$

In this form, the slopes of the asymptotes are readily seen to be $-b_{1} / a_{1}$ and $-b_{2} / a_{2}$.

From equation (34), the two eigenvalues $\bar{\omega}_{n, m}$ in the veering or flutter region of the weakly coupled system $(0<\varepsilon \ll 1)$,

$$
\bar{\omega}_{n, m}=\omega_{1}+\left[-\left(a_{1} b_{2}-a_{2} b_{1}\right)\left(\bar{\eta}-\eta_{1}\right) \pm \sqrt{\left(a_{1} b_{2}+a_{2} b_{1}\right)^{2}\left(\bar{\eta}-\eta_{1}\right)^{2}+4 a_{1} a_{2} \Lambda}\right] / 2 a_{1} a_{2},
$$


are known functions of the new speed parameter $\bar{\eta}$ and the eigenvalues $\omega^{*}$ of the decoupled system $(\varepsilon=0)$. Moreover, the eigenvalues of the decoupled system are already known from equations (24) and (25). At $\bar{\eta}=\eta_{1}$, the approximation gives

$$
\bar{\omega}_{n, m}\left(\bar{\eta}=\eta_{1}\right)=\omega_{1} \pm \sqrt{Q},
$$

where $Q$, which may be positive, negative or zero, is given by

$$
Q=p^{*} q^{*} / a_{1} a_{2}=\omega^{* 2}\left(\gamma^{2}-\eta^{* 2}\right)\left(1-\eta^{* 2}\right) / d(1-d) .
$$

Thus, the sign of $Q$ determines whether the perturbed eigenvalues are real or complex and therefore whether the loci crossing evolves into a region of curve veering $(Q>0)$ or a region of flutter instability $(Q<0)$. The following three possibilities are noted from equation (37): (i) when $Q>0$ for $0<\eta^{*}<\gamma$ or $\eta^{*}>1$, two real and distinct eigenvalues result, and a region of curve veering develops; (ii) when $Q=0$ for $\eta^{*}=\gamma$ or 1 , repeated (zero) eigenvalues remain; (iii) when $Q<0$ for $\gamma<\eta^{*}<1$, two complex conjugate eigenvalues result, and a region of flutter instability develops.

The accuracy of this approximation is demonstrated in Figure 6, where the perturbation solution (dashed curve) is compared to the exact solution (solid curve) for the regions of curve veering and flutter instability shown by the boxes in Figure 3 . The dotted lines represent the asymptotes of the hyperbola. The perturbation solution remains within $2 \cdot 5 \%$ of the exact solution over the ranges of $\eta$ shown in Figure 6. Note that the perturbation solution permits the regions of flutter instability to be determined without numerical computation of the eigenvalues from equation (20). From (36), the flutter region is easily found by identifying the speeds $\bar{\eta}$ for which $\bar{\omega}_{n}=\bar{\omega}_{m}$.

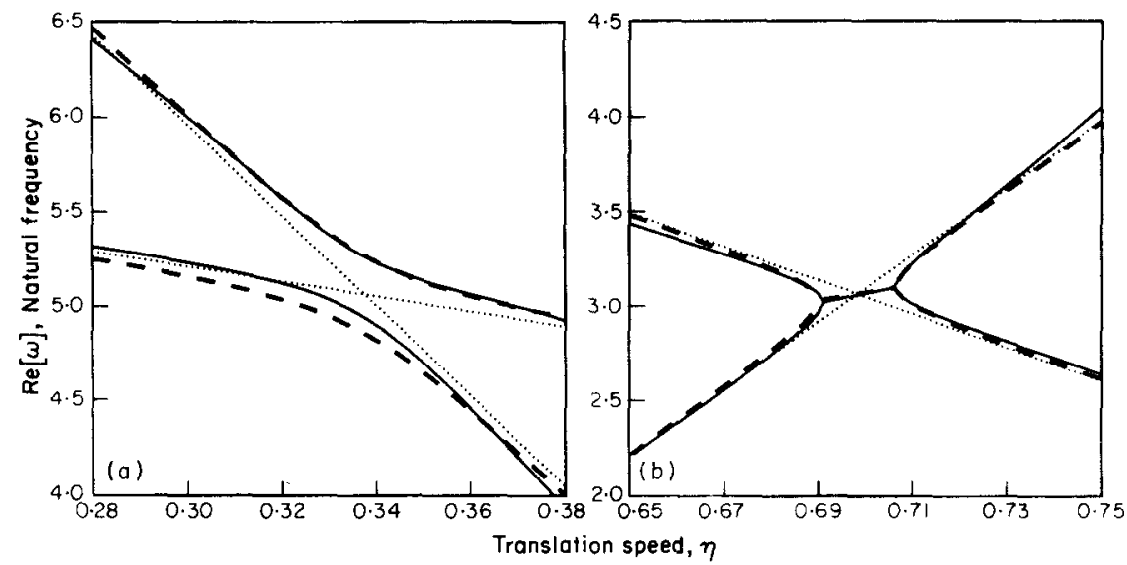

Figure 6. Perturbation solution for representative regions of curve veering (a) and flutter instability (b) in Figure 3. Solid curves are the exact solution; dashed curves are the perturbation solution. Dotted lines represent the asymptotes of the hyperbola.

This perturbation solution demonstrates that regions of curve veering and flutter instability are naturally described by a local hyperbolic approximation. This may, in part, explain the inadequacy of the classical, parabolic approximation for curve veering, previously noted in reference [13]. Note that, in this problem, both types of regions evolve from regions of loci crossings $(k \rightarrow \infty)$, which are described by the degenerate form of the hyperbola, $\Lambda=0$ in equation (34). 


\section{RESPONSE TO HARMONIC END EXCITATION}

Mechanical winders often impart a lateral motion to the translating element and represent an important source of external excitation. Here, the forced response of the string/guide system to harmonic excitation applied at the upstream end is examined.

In this case, the equations of motion $(7)-(10)$ are solved subject to the boundary conditions

$$
U_{1}(0, T)=Q \cos \Omega_{f} T, \quad U_{2}(L, T)=0 .
$$

The introduction of additional non-dimensional variables, $u_{1}=U_{1} / L, u_{2}=U_{2} / L, q=Q / L$, $\omega_{f}=\Omega_{f} L / c$, enables the steady state response of the system to be written as

$$
\begin{aligned}
u_{1}(x, t)= & q \cos \left(\omega_{f} t+\phi_{1} x\right)\left\{\cos \left(\psi_{1} x\right)+\left[\sin \left(\psi_{1} x\right) / C\left(\omega_{f}\right)\right]\left[-\omega_{f} \cos \left(\psi_{1} d\right) \cos \left(\psi_{2}-\psi_{2} d\right)\right.\right. \\
& \left.\left.+\gamma \omega_{f} \sin \left(\psi_{1} d\right) \sin \left(\psi_{2}-\psi_{2} d\right)-k \cos \left(\psi_{1} d\right) \sin \left(\psi_{2}-\psi_{2} d\right)\right]\right\} \\
u_{2}(x, t)= & q \cos \left[\omega_{f} t+\phi_{2} x+\left(\phi_{1}-\phi_{2}\right) d\right] \\
& \times\left\{-\left[\gamma \omega_{f} \cos \left(\psi_{2}\right) / C\left(\omega_{f}\right)\right]\left[\sin \left(\psi_{2} x\right)-\tan \left(\psi_{2}\right) \cos \left(\psi_{2} x\right)\right]\right\}
\end{aligned}
$$

Note that the factors in curly brackets in equations (40) and (41) describe the amplitude of the forced response envelope which becomes unbounded under the resonance condition $C\left(\omega_{f}=\omega_{n}\right)=0$. Near this resonance condition, $\omega_{f} \simeq \omega_{n}$, the response envelope closely resembles that of the free response associated with the natural frequency $\omega_{n}$. Moreover, if this natural frequency lies in a region of curve veering, then large changes in the forced response envelope follow from small changes in operating conditions.

The sensitivity of the forced response is illustrated in Figure 7, which shows the forced response envelope for three sets of close operating conditions. In this example, the guide stiffness, friction and translation speed are all held constant. The dramatic changes observed in the response derive from small changes in either the guide location $d$ or the excitation frequency $\omega_{f}$. The three sets of values chosen for $d$ and $\omega_{f}$ are defined by the points $\mathbf{a}, \mathbf{b}$ and $\mathbf{c}$ in Figure 5, near the region of curve veering for the first two modes. The solid curve in Figure 7 represents a response that is localized to the downstream portion of the system for an excitation frequency slightly lower than the fundamental natural frequency (corresponds to $a$ in Figure 5). The forced response becomes localized to the upstream portion of the system after the guide location is adjusted from $d=0 \cdot 2$ (point a) to $d=0.277$ (point b); see the dashed curve in Figure 7. A similar change in

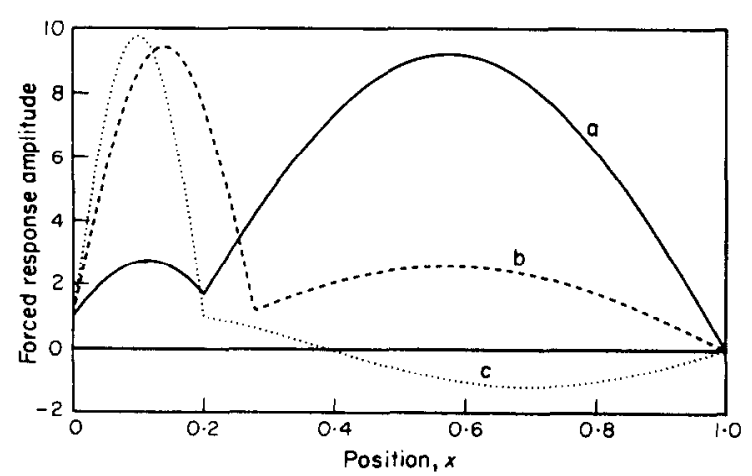

Figure 7. Localization of forced response for three close operating conditions defined by $\mathbf{a}, \mathbf{b}$ and $\mathbf{c}$ points in Figure 5. Solid curve: point $a, d=0.2$ and $\omega_{j}=3.36$; dashed curve: point $b, d=0.277$ and $\omega_{f}=3.36$; dotted curve: point $c, d=0.2$ and $\omega_{f}=4 \cdot 70$. In all cases, $\eta=0.3, \gamma=0.5$ and $k=20$. 
the response could also be produced by changing the excitation frequency to a value close to the second natural frequency (point c); see the dotted curve in Figure 7. This result serves to illustrate how the forced response may be localized to either segment of the system by small adjustments to the operating conditions.

\section{CONCLUSIONS}

Exact expressions have been derived for the free and forced linear response of a translating string coupled to a dry friction guide. Solutions for the eigenvalue spectrum illustrate how the vibration and stability of the string/guide system can be modified by adjusting the design variables such as guide friction, location, stiffness, translation speed and tension. In particular, the range of stable operating speeds can be increased by either reducing the guide friction or increasing the prescribed downstream tension. For large values of the guide stiffness, the frequency spectrum in the stable range of speeds contains regions of curve veering, and the free response becomes highly localized to the upstream or downstream segments. The location (upstream or downstream) of the maximum response under forced conditions, therefore, may be strongly influenced by the selection of design variables. A derived perturbation solution greatly facilitates this selection by providing a closed form approximation for the eigenvalue spectrum in the regions of curve veering. The perturbation solution also highlights the common features of the regions of curve veering and the regions of flutter instability observed in this system. In both regions, the eigenvalue loci are naturally described by a local hyperbolic approximation.

\section{ACKNOWLEDGMENT}

The authors gratefully acknowledge the partial support of the U.S. Office of Naval Research.

\section{REFERENCES}

1. J. A. WICKert and C. D. MOTE, JR. 1988 Shock and Vibration Digest 20, 3-13. Current research on the vibration and stability of axially moving materials.

2. R. D. SWOPE and W. F. AMES 1963 Journal of the Franklin Institute 275, 36-55. Vibrations of a moving threadline.

3. G. S. SCHAJER 1984 Journal of Sound and Vibration 92, 11-19. The vibration of a rotating circular string subject to a fixed end restraint.

4. N. C. Perkins and C. D. MOTE, JR. 1986 Journal of Sound and Vibration 106, 451-463. Comments on curve veering in eigenvalue problems.

5. R. B. BHAT, G. D. XISTRIS and T. S. SANKAR 1982 American Society of Mechanical Engineers Journal of Mechanical Design 104, 143-147. Dynamic behavior of a moving belt supported on elastic foundation.

6. N. C. PERKINS 1990 American Society of Mechanical Engineers Journal of Vibration and Acoustics 112, 2-7. Linear dynamics of a translating string on an elastic foundation.

7. C. D. MOTE, JR. 1966 American Society of Mechanical Engineers Journal of Applied Mechanics 33, 463-464. On the nonlinear oscillation of an axially moving string.

8. A. G. Ulsoy, J. E. Whitesell and M. D. HoOVEN 1985 American Society of Mechanical Engineers Journal of Vibration, Acoustics, Stress and Reliability in Design 107, 282-290. Design of belt-tensioner systems for dynamic stability.

9. J. J. THWAITES 1970 Journal of the Textile Institution 61, 116-138. The mechanics of frictiontwisting.

10. K. ONO 1979 American Society of Mechanical Engineers Journal of Applied Mechanics 46, 905-912. Lateral motion of an axially moving string on a cylindrical guide surface. 
11. T. MAJEWSKI 1986 Journal of Sound and Vibration 105, 17-25. Audio signal modulation caused by self-excited vibrations of magnetic tape.

12. C. Pier Re and E. H. Dowell 1987 Journal of Sound and Vibration 114, 549-564. Localization of vibrations by structural irregularity.

13. C. PIERRE 1988 Journal of Sound and Vibration 126, 485-502. Mode localization and eigenvalue loci veering phenomena in disordered structures. 